\section{Gut epithelial barrier markers in patients with obstructive sleep apnea}

Antonia Barceló ${ }^{\text {a,d,ff, }, * \text {, Cristina Esquinas }}{ }^{\mathrm{f}}$, Juan Robles ${ }^{\text {a }}$, Javier Piérola ${ }^{\mathrm{c}, \mathrm{d}}$, Mónica De la Peña ${ }^{\text {b,d,f }}$, Irene Aguilar ${ }^{\text {a }}$, Daniel Morell-Garcia ${ }^{a}$, Alberto Alonso ${ }^{\text {b,d,f }}$, Nuria Toledo ${ }^{\text {b }}$, Manuel Sánchez-de la Torre e,f , Ferran Barbé e,f

${ }^{a}$ Servei de Anàlisis Clíniques, Hospital Universitari Son Espases, Palma de Mallorca, Spain

${ }^{\mathrm{b}}$ Servei de Pneumologia, Hospital Universitari Son Espases, Palma de Mallorca, Spain

${ }^{c}$ Servei de Unitat de Investigació, Hospital Universitari Son Espases, Palma de Mallorca, Spain

${ }^{\mathrm{d}}$ Hospital Universitari Son Espases and Institut de investigacio (IdISPa), Palma de Mallorca, Spain

${ }^{\mathrm{e}}$ Respiratory Department, Hospital Universitari Arnau de Vilanova and Santa Maria, Lleida, IRBLleida, Lleida, Catalonia, Spain

${ }^{\mathrm{f}}$ Centro de Investigación Biomédica en Red de Enfermedades Respiratorias (CIBERES) Spain.

*Corresponding author: Servei de Anàlisis Clíniques, Hospital Universitari Son Espases, Crta. Valldemossa 79, 07120, Palma de Mallorca, Spain. Tel.: 0034871205000(75876); fax: 0034871909706.

E-mail address: antonia.barcelo@ssib.es (A, Barceló)
Comment [JM1]: Would it be better to use people instead of patients? It is a bit more generic.

Comment [JM2]: Authors: affiliations are usually in alphabetical order, so these three would be a,b,c and so on. Please check your affiliation letters and amend and re-order your affiliations to match. Please also check all of your affiliations addresses, as this journal requires department, institution, city and country to be listed for each affiliation. Thanks. 


\section{HIGHLIGHTS}

- The intestinal wall integrity plays a role in the development of metabolic disorders.

- The influence of obstructive sleep apnea (OSA) on markers of gut barrier (fatty acid binding protein (I-FABP) and Zonulin) was investigated.

- The I-FABP levels were higher in the presence of OSA.

- Zonulin levels were associated with metabolic parameters.

- Obstructive sleep apnea may be a risk factor for intestinal damage, regardless of metabolic profile.

\section{Abstract}

Background: Obstructive sleep apnea (OSA) is now being recognized as an additional contributing factor to the pathogenesis of obesity-related comorbidities. At the same time, there is now increasing evidence to suggest that intestinal wall integrity plays a role in the development of metabolic syndrome. In the present study, circulating zonulin and fatty acid binding protein (I-FABP) were measured in association with metabolic, hepatic and inflammatory parameters.

Results: Compared with controls, plasma I-FABP levels were significantly higher in patients with OSA (571 pg/mL (IQR 290-950) vs 396 pg/mL (IQR 234-559), p=0.04). zonulin levels were similar between groups. Significant relationships were observed between zonulin levels and waist circumference $(p<0.05)$, glucose $(p<0.05)$ and insulin $(p<0.05)$. In addition, in the OSA group, zonulin levels correlated negatively with the mean nocturnal oxygenation saturation $(p<0.05)$ and positively with total cholesterol $(p<0.05)$, alanine aminotransferase (ALT) $(p<0.005)$, aminotransferase (AST) $(p<0.01)$, gamma glutamyltransferase (GGT) $(p<0.005)$ and high-sensitivity C-reactive protein (hs-CRP) $(p<0.05)$. Multivariate analysis showed that associations between zonulin and ALT, AST and hs-CRP were attenuated, but not eliminated, after adjustment for other variables.

Conclusions: The results of this study suggest that OSA is a risk factor for intestinal damage, regardless of metabolic profile, and that intestinal permeability might be a possible contributor to nonalcoholic fatty liver disease in patients with OSA. 


\section{Keywords:}

Zonulin

Fatty acid binding protein (I-FABP)

Intestinal wall

Sleep apnea

\section{Introduction}

Obstructive sleep apnea syndrome (OSA) is a common disorder that is defined by the occurrence of repeated episodes of upper airway obstruction and airflow cessation (apneas) that normally lead to intermittent arterial hypoxemia (IH) and sleep disruption [1,2]. The effects of these cyclical changes of hypoxemia with reoxygenation are similar to ischemiareperfusion injury, and evidence from both animal and human studies have shown a predominant role of IH in the pathogenesis of OSA comorbidities [3,4].

Recently, there has been a lot of interest in the role of intestinal wall integrity and gut microbiota in the pathogenesis of obesity and its concomitant disease [5,7]. Loss of intestinal barrier function or changes in gut microbiota both seem to be key ingredients involved in the pathogenesis of metabolic disorders [8-10]. In the present study, it was hypothesized that OSA may affect the intestinal barrier function by altering structure and permeability. Repetitive periods of intestinal ischemia reperfusion could result in severe damage to the intestinal barrier, thereby allowing translocation of luminal antigens, bacteria, and their toxic products from the intestinal lumen to the systemic circulation. In addition, persistently increased permeability may be an important contributor to the development of metabolic complications. To test this hypothesis a case-control study was performed and two novel gut barrier markers were evaluated: intestinal fatty acid binding protein (I-FABP) and zonulin. 
Plasma I-FABP is a highly sensitive marker for intestinal ischemia. Intestinal fatty acid binding proteins are small proteins released into the circulation upon enterocyte membrane integrity loss, which makes them useful as plasma markers for enterocyte damage during the development and progression of intestinal ischemia-reperfusion [11-13]. By contrast, zonulin is expressed by viable gut epithelial cells to disassemble tight junctions between cells, and increase permeability and macromolecule absorption. Previous studies have claimed a role for zonulin and its regulation of intestinal barrier function in the genesis of metabolic disorders $[10,14,15]$.

\section{Methods}

\section{Subjects and ethics}

Patients and controls were recruited from subjects who attended the present sleep unit during a period of 2 years. In the present study, two subgroups (patients and controls) of participants were retrospectively selected. The final sample comprised 38 patients with OSA and 38 controls matched for sex, age ( \pm 5 years), body mass index (BMI) $( \pm 3 \mathrm{~kg} / \mathrm{m})$ and the presence of metabolic syndrome.

No participant was under continuous positive airway pressure (CPAP) treatment or suffered from any other chronic disease (chronic obstructive pulmonary disease (COPD), liver cirrhosis, thyroid dysfunction, rheumatoid arthritis, coronary disease, cardiac failure, chronic renal failure and/or psychiatric disorders). There were no differences between the number of patients and controls taking hypoglycemic, hypolipemiant and/or antihypertensive agents. No participant was regularly taking anti-inflammatory medication. The study was approved by the Ethics Committee of the present institution, and all participants signed their consent after being fully informed of its goals and characteristics. 


\section{Measurements and definitions}

The diagnosis of OSA was established by full polysomnography (E-Series Compumedics, Abbotsford, Australia) that included recording of oronasal flow, thoracoabdominal movements, electrocardiography, submental and pretibial electromyography, electrooculography, electroencephalography and transcutaneous measurement of arterial oxygen saturation. Apnea was defined by the absence of airflow for $>10$ seconds. Hypopnea was defined as any airflow reduction that lasted $>10$ seconds and resulted in arousal or oxygen desaturation. Desaturation was considered to be a decrease in $\mathrm{SaO}_{2}>3 \%$. The apneahypopnea index (AHI) was defined as the sum of the number of apneas plus hypopneas per hour of sleep. The case or control status was defined by an AHI threshold of $\geq 10$. Excessive daytime sleepiness (EDS) was subjectively quantified by the Epworth sleepiness scale (ESS).

The occurrence of the metabolic syndrome was analyzed according to the National Cholesterol Education Program Adult Treatment Panel III (NCEP ATP III ) clinical criteria [16]: (1) waist circumference $\geq 102 \mathrm{~cm}$ in men and $\geq 88 \mathrm{~cm}$ in women; (2) fasting glucose $\geq 100 \mathrm{mg} / \mathrm{dL}$; (3) triglycerides $\geq 150 \mathrm{mg} / \mathrm{dL}$; (4) HDL cholesterol (HDLc) $<40 \mathrm{mg} / \mathrm{dL}$ in men and $<50 \mathrm{mg} / \mathrm{dL}$ in women; (5) systolic blood pressure (SBP) $\geq 130 \mathrm{mmHg}$ or diastolic blood pressure (DBP) $\geq 85 \mathrm{mmHg}$ or patient on specific drug treatment. Metabolic syndrome was diagnosed if three of these five factors were present. Diabetes was defined by use of diabetes medications or a fasting glucose $\geq 126 \mathrm{mg} / \mathrm{mL}$. Hypertension was diagnosed if the SBP was $\geq 140 \mathrm{mmHg}$ and/or DBP was $\geq 90 \mathrm{mmHg}$ or the individual was on specific treatment. Participants were considered obese when their BMI was $\geq 30 \mathrm{~kg} / \mathrm{m}$. 
After fasting overnight, venous blood samples were obtained between 08:00 and 10:00. Blood was centrifuged and serum was immediately separated in aliquots and stored at $-80{ }^{\circ} \mathrm{C}$ until analysis.

Glucose, triglycerides, total cholesterol, HDLc, creatinine, aspartate aminotransferase (AST), alanine aminotransferase (ALT) and gamma glutamyltransferase (GGT) were determined by standard enzymatic methods on a Hitachi Modular analyzer (Roche Diagnostics, Indianapolis, USA). The high-sensitivity C-reactive protein (hs-CRP) assay was performed using a chemiluminescence assay on an Immulite 2000 analyzer (Siemens Medical Solutions Diagnostics, NY, USA).

The plasma levels of zonulin and I-FABP were determined by enzyme linked immunosorbent assay (ELISA) using commercial kits (zonulin: Immunodiagnostik AG, Bensheim, Germany; I-FABP: Hycult Biotechnology, Uden, The Netherlands). Measurements were always conducted in duplicate, and mean values were used for analysis. The detection limit for zonulin was $0.22 \mathrm{ng} / \mathrm{mL}$ and for I-FABP $40 \mathrm{pg} / \mathrm{mL}$. Intra and interassay coefficients of variation were between $3-10 \%$.

\section{Statistical analysis}

Results were presented as percentages, mean \pm standard deviations (SD) or median (interquartile range). Skewed variables were logarithmically transformed before analysis Patients characteristics were compared using independent sample $t$-tests for continuous measures or repeated measures ANOVA, when appropriate. Categorical variables were compared using the Chi-squared or Fisher tests. 
Correlations between the subjects' characteristics and zonulin and I-FABP were explored using the Spearman-rank test. Multiple regression analyses were used to confirm the significant associations detected with adjustment for age, sex, BMI and metabolic syndrome components.

The study was powered not a miss a difference of $1.5 \mathrm{ng} / \mathrm{mL}$ in zonulin, assuming a SD of

$2 \mathrm{ng} / \mathrm{mL}$ in healthy subjects [14] at a significance level of $5 \%$ and with a power of $90 \%$, which required 31 subjects in each group. A $p$-value $<0.05$ was considered significant.

\section{Results}

Thirty-eight patients with OSA and 38 control subjects were studied. The main clinical and biochemical characteristics are shown in Table 1. By design, characteristics of patients, including age, sex, BMI and the prevalence of metabolic syndrome, were similar between patients and controls.

Compared with controls subjects, the OSA patients showed elevated plasma levels of I-FABP

(Table 1). Participants were divided into three tertiles based on their AHI (AHI <7.5, $n=25$; AHI 7.6-28.5, $n=26$; AHI >28.6, $n=25$ ). There was a significant trend of increasing I-FABP levels with increasing AHI ( $\mathrm{p}_{\text {trend }}$ 0.049) (Fig. 1).

Multivariate analysis showed that there were no significant associations between

I-FABP levels and indices of nocturnal hypoxia (mean and minimal nocturnal oxygenation saturation) after adjustment for other variables. No significant differences in zonulin levels were detected between OSA patients and controls (Table 1). 
Significant associations between zonulin levels and waist circumference, glucose and insulin were detected both in controls and patients with OSA. In addition, in the OSA group, zonulin levels correlated negatively with the mean nocturnal oxygenation saturation and positively with total cholesterol, ALT, AST, GGT and hs-CRP (Table 2). The associations between zonulin and ALT, AST and hs-CRP were attenuated, but not eliminated, after adjustment for age, sex, BMI and components of metabolic syndrome in multivariate analysis (Table 3 ).

\section{Discussion}

This study showed that: (1) the I-FABP levels were elevated in patients with OSA, suggesting that OSA itself is a risk factor for intestinal damage; (2) the zonulin levels were associated with the presence of obesity and metabolic disturbances both in OSA and nonOSA patients; (3) among the OSA patients, zonulin levels were positively correlated with aminotransferases levels, suggesting a possible link between intestinal permeability and nonalcoholic fatty liver disease (NAFLD).

Intestinal fatty acid binding proteins are small cytosolic proteins present in mature enterocytes and they are released into the circulation upon membrane integrity loss $[6,17]$. Previous studies have reported on the value of I-FABP as a biomarker for intestinal ischemia, and a relationship between plasma I-FABP levels and the degree of ischemic periods has been detected [18]. In the present study, despite the similar anthropometric and metabolic characteristics between the OSA participants and controls, the I-FABP levels were higher in the OSA group and tended to increase with an increasing AHI, suggesting an intestinal epithelial injury in these patients. The cyclical changes of hypoxemia with reoxygenation could represent an important mechanism for the presence of intestinal damage in OSA, independent of metabolic disturbances. Disruption of the gut wall integrity and leakage of 
macromolecules and microbial products from the intestinal lumen into the circulation can induce local and systemic inflammatory responses [19-21]. The present results suggest that OSA might contribute to gut mucosal injury, which might have important clinical implications. Assessment of plasma I-FABP offers the possibility to monitor the presence of intestinal ischemia, and several studies have shown their clinical usefulness as a marker for epithelial intestinal damage and their prognostic value for adverse outcomes $[6,11-13,17]$.

In the present study, it was also observed that the zonulin levels were not significantly different between patients with OSA and controls without OSA of similar metabolic profile. Recent studies have suggested a role for increased intestinal permeability in the genesis of obesity and metabolic disorders $[6,14,22]$. Zonulin regulates intestinal permeability by modulating intercellular tight junctions, and higher zonulin levels have been detected in the obese and those with impaired glucose tolerance [8,23-25]. In the present study, circulating zonulin concentrations were correlated with obesity-related disturbances (waist circumference, glucose, insulin and total cholesterol) both in OSA and non-OSA subjects. These results suggest that sleep apnea does not have any direct effect on the circulating zonulin concentrations. Nevertheless, although the present results suggest that the effects on zonulin concentrations and intestine permeability may be dependent on obesity index or metabolic dysfunction, rather than on sleep apnea by itself, does not mean that they are irrelevant in the pathogenesis of several complications in these patients. There is now evidence that interactions between gut microbiota and other intestinal factors are emerging mechanisms influencing the onset of NAFLD [26]. In this sense, a recent study has shown that in subjects with NAFLD, circulating zonulin was increased in association with the severity of steatosis, providing a possible link between intestinal permeability and the development of NAFLD [15]. In parallel with these studies, evidence is growing that OSA is 
an independent factor for NAFLD [27,28]. The present study showed that among OSA patients, zonulin was also associated with aminotransferases (AST, ALT) and GGT, which are the main tests used to assess liver injury and surrogate markers of NAFLD, the hepatic manifestation of metabolic syndrome. These observations suggest a potential role of the zonulin system in the link between OSA and liver injury, raising the possibility that the influence of sleep apnea on liver dysfunction liver may particularly vary as a function of their degree of intestine permeability independently of obesity or metabolic conditions.

The strengths of this study included the assessment of associations between circulating concentrations of I-FABP (marker of gut ischemia) and zonulin (marker and modulator of intestinal permeability) with OSA and the presence of metabolic syndrome. Taken together, the present findings suggest that the effects of OSA on gut barrier markers (I-FABP and zonulin) may represent different underlying pathways, with OSA likely acting through ischemic periods, and gut mucosal damage and the zonulin system having a more important effect on the liver consequences via intestinal permeability.

Several limitations should be considered. First, the main limitation was the relatively small number of participants. Despite the study design and the metabolic similarity between OSA and controls, the lack of power to detect differences between these two groups cannot be excluded. The observation of a non-significant trend of an independent association between IFABP levels and indices of nocturnal hypoxia may have been due to the lack of power to detect this difference. Second, although AHI reflects OSA severity, an additional estimation of the oxygen desaturation index could have reinforced the relative role of OSA on gut barrier integrity markers. Third, the cross sectional nature of the study did not prove causeeffect relationships between OSA and gut barrier markers. Finally, the plasma levels of I- 
FABP and zonulin after CPAP were not measured, and this may have been a limitation in the assessment of the independent effects of OSA on these markers.

\section{Conclusions}

The results of this study showed that I-FABP levels were elevated in patients with OSA, suggesting that OSA itself is a risk factor for intestinal damage. Zonulin levels were associated with the presence of obesity and metabolic disturbances both in OSA and nonOSA patients. In addition, among the OSA patients, zonulin levels were positively correlated with aminotransferases levels, suggesting a possible link between intestinal permeability and nonalcoholic fatty liver disease.

Conflict of interest: All authors declare no conflicts of interest.

Funding statement: This study was supported in part by Fondo de Investigaciones Sanitarias.

Mention of any meeting(s) where the information has previously been presented: The manuscript content (in part or in full) has not been presented elsewhere. 


\section{References}

1. Young T, Peppard PE, Gottlieb DJ. Epidemiology of obstructive sleep apnea: a population health perspective. Am J Respir Crit Care Med 2002;165:1217-39.

2. Douglas NJ, Polo O. Pathogenesis of sleep apnoea/hypopnoea syndrome. Lancet 1994;344:653-5.

3. Dewan NA, Nieto FJ, Somers VK. Intermittent hypoxemia and OSA: implications for comorbidities. Chest 2015;147:266-74.

4. Ong CW, O'Driscoll DM, Truby H, Naughton MT, Hamilton GS. The reciprocal interaction between obesity and obstructive sleep apnoea. Sleep Med Rev 2013;17:12331.

5. Sekirov I, Russell SL, Antunes LC, Finlay BB. Gut microbiota in health and disease. Physiol Rev 2010;90:859-904.

6. Derikx JP, Luyer MD, Heineman E, Buurman WA. Non-invasive markers of gut wall integrity in health and disease. World J. Gastroenterol 2010;16:5272-9.

7. Vajro P, Paolella G, Fasano A. Microbiota and gut-liver axis: their influences on obesity and obesity-related liver disease. J Pediatr Gastroenterol Nutr 2013;56:461-8.

8. Moreno-Navarrete JM, Sabater M, Ortega F, Ricart W, Fernandez-Real JM. Circulating zonulin, a marker of intestinal permeability, is increased in association with obesityassociated insulin resistance. PLoS One 2012;7: e37160. 
9. Fasano A. Intestinal permeability and its regulation by zonulin: diagnostic and therapeutic implications. Clin Gastroenterol Hepatol 2012;10:1096-100.

10. Sapone A, de Magistris L, Pietzak M, et al. zonulin upregulation is associated with increased gut permeability in subjects with type 1 diabetes and their relatives. Diabetes 2006;55:1443-9.

11. Schellekens DH, Grootjans J, Dello SA, et al. Plasma intestinal fatty acid-binding protein levels correlate with morphologic epithelial intestinal damage in a human translational ischemia-reperfusion model. J Clin Gastroenterol 2014;48:253-60.

12. Derikx JP, Matthijsen RA, de Bruine AP, et al. Rapid reversal of human intestinal ischemia-reperfusion induced damage by shedding of injured enterocytes and reepithelialisation. PLoS One 2008;3:e3428.

13. Thuijls G, van Wijck K, Grootjans J, et al. Early diagnosis of intestinal ischemia using urinary and plasma fatty acid binding proteins. Ann Surg 2011;253:303-8.

14. Zak-Golab A, Kocelak P, Aptekorz M, et al. Gut microbiota, microinflammation, metabolic profile, and zonulin concentration in obese and normal weight subjects. Int $\mathrm{J}$ Endocrinol 2013;2013:674106.

15. Pacifico L, Bonci E, Marandola L, Romaggioli S, Bascetta S, Chiesa C. Increased circulating zonulin in children with biopsy-proven nonalcoholic fatty liver disease. World J Gastroenterol 2014;20:17107-14. 
16. Alberti KG, Eckel RH, Grundy SM, et al. Harmonizing the metabolic syndrome: a joint interim statement of the International Diabetes Federation Task Force on Epidemiology and Prevention; National Heart, Lung, and Blood Institute; American Heart Association; World Heart Federation; International Atherosclerosis Society; and International Association for the Study of Obesity. Circulation 2009;120:1640-5.

17. Reisinger KW, Elst M, Derikx JP, et al. Intestinal fatty acid-binding protein: a possible marker for gut maturation. Pediatr Res 2014;76:261-8.

18. Derikx JP, Vreugdenhil AC, Van den Neucker AM, et al. A pilot study on the noninvasive evaluation of intestinal damage in celiac disease using I-FABP and LFABP. J Clin Gastroenterol 2009;43:727-33.

19. Grootjans J., Thuijls G, Verdam F, Derikx JP, Lenaerts K, Buurman WA. Non-invasive assessment of barrier integrity and function of the human gut. World J Gastrointest Surg 2010;2:61-9.

20. Somsouk M, Estes JD, Deleage C, et al. Gut epithelial barrier and systemic inflammation during chronic HIV infection. AIDS 2015;29:43-51.

21. Schurink M, Scholten IG, Kooi EM, et al. Intestinal fatty acid-binding protein in neonates with imminent necrotizing enterocolitis. Neonatology 2014;106:49-54.

22. Rapin JR, Wiernsperger N. Possible links between intestinal permeability and food processing: A potential therapeutic niche for glutamine. Clinics (Sao Paulo) 2010;65:635-43. 
23. Fasano A. Physiological, pathological, and therapeutic implications of zonulin-mediated intestinal barrier modulation: living life on the edge of the wall. Am J Pathol 2008;173:1243-52.

24. Zhang D, Zhang L, Yue F, Zheng Y, Russell R. Serum zonulin is elevated in women with polycystic ovary syndrome and correlates with insulin resistance and severity of anovulation. Eur J Endocrinol 2015;172:29-36.

25. Zhang D, Zhang L, Zheng Y, Yue F, Russell RD, Zeng Y. Circulating zonulin levels in newly diagnosed Chinese type 2 diabetes patients. Diabetes Res Clin Pract 2014;106:312-8.

26. Compare D, Coccoli P, Rocco A, Nardone OM, De Maria S, Carteni M, et al. Gut--liver axis: the impact of gut microbiota on non alcoholic fatty liver disease. Nutr Metab Cardiovasc Dis 2012;22:471-6.

27. Minville C, Hilleret MN, Tamisier R, et al. Nonalcoholic fatty liver disease, nocturnal hypoxia, and endothelial function in patients with sleep apnea. Chest 2014;145:525-33.

28. Moreno-Indias I, Torres M, Montserrat JM, et al. Intermittent hypoxia alters gut microbiota diversity in a mouse model of sleep apnoea. Eur Respir J 2015;45:1055-65. 


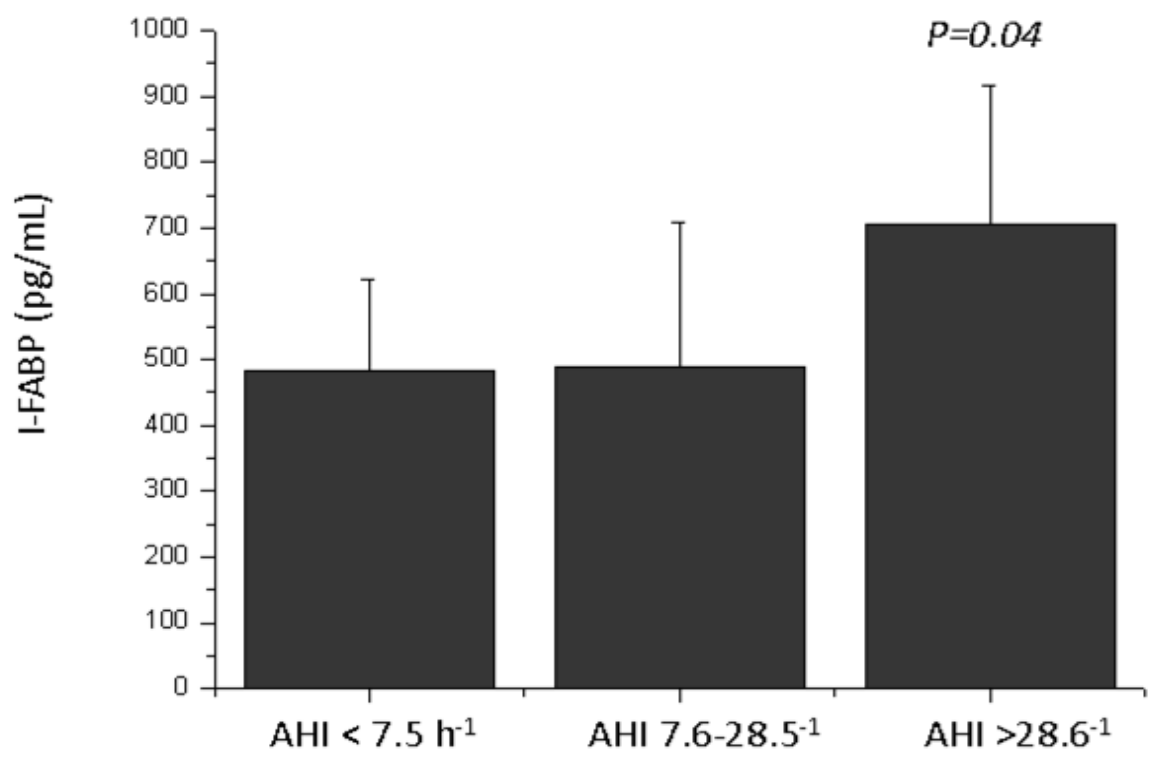

Fig. 1. I-FABP levels according to the apnea-hypopnea index (AHI).

Comment [JM9]: Please see edited version of

this as the units need to be amended: This

journal requires SI unit symbols with a / not -1

Table 1. Baseline characteristics and sleep profiles in controls and OSA patients.

\begin{tabular}{|l|c|c|}
\hline & $\begin{array}{c}\text { Controls } \\
(n=38)\end{array}$ & $\begin{array}{c}\text { OSA } \\
(n=38)\end{array}$ \\
\hline Age (years) & $49 \pm 12$ & $50 \pm 12$ \\
\hline Men (\%) & $19(50 \%)$ & $21(52 \%)$ \\
\hline BMI $(\mathrm{kg} / \mathrm{m})$ & $29 \pm 6$ & $28 \pm 6$ \\
\hline Waist circumference (cm) & $96 \pm 13$ & $95 \pm 12$ \\
\hline Hypertension $(\%)$ & $4(11 \%)$ & $12(32 \%)^{*}$ \\
\hline Diabetes $(\%)$ & $2(5 \%)$ & $14(37 \%)$ \\
\hline Metabolic syndrome $(\%)$ & $14(37 \%)$ & $2.2 \pm 1.4$ \\
\hline Number of metabolic syndrome & $2.1 \pm 1.3$ & \\
\hline criteria $(\%)$ & & $45.5 \pm 29 *$ \\
\hline AHI (hour $\left.{ }^{-1}\right)$ & $5.7 \pm 2.6$ & $93 \pm 4 *$ \\
\hline Mean SaO ${ }_{2}(\%)$ & $95 \pm 2$ & \\
\hline
\end{tabular}

Comment [JM10]: Please list all abbreviations in footnotes

\section{Comment [JM11]: As before}

Comment [JM12]: This journal requires $\mathrm{SI}$ unit symbols with a / not -1 


\begin{tabular}{|l|c|c|}
\hline Minimal $\mathrm{SaO}_{2}(\%)$ & $90 \pm 4$ & $82 \pm 9 *$ \\
\hline Glucose $(\mathrm{mg} / \mathrm{dL})$ & $99 \pm 17$ & $98 \pm 7$ \\
\hline Triglycerides $(\mathrm{mg} / \mathrm{dL})$ & $127 \pm 48$ & $133 \pm 44$ \\
\hline Cholesterol $(\mathrm{mg} / \mathrm{dL})$ & $199 \pm 41$ & $200 \pm 47$ \\
\hline HDLc $(\mathrm{mg} / \mathrm{dL})$ & $54 \pm 17$ & $0.85 \pm 0.2$ \\
\hline Creatinine $(\mathrm{mg} / \mathrm{dL})$ & $0.87 \pm 0.2$ & $9.9 \pm 7.2$ \\
\hline Insulin $(\mu \mathrm{UI} / \mathrm{mL})$ & $9.6 \pm 6.5$ & $21(17-25)$ \\
\hline AST $(\mathrm{U} / \mathrm{L})$ & $19(16-22)$ & $23(16-29)$ \\
\hline ALT $(\mathrm{U} / \mathrm{L})$ & $21(14-27)$ & $21(14-38)$ \\
\hline GGT $(\mathrm{U} / \mathrm{L})$ & & $0.2(0.1-0.5)$ \\
\hline hs-CRP $(\mathrm{mg} / \mathrm{L})$ & $22(13-37)$ & $4.2(3.3-4.9)$ \\
\hline zonulin $(\mathrm{ng} / \mathrm{mL})$ & $0.2(0.1-0.4)$ & $571(290-950)^{*}$ \\
\hline I-FBAP $(\mathrm{pg} / \mathrm{mL})$ & $4.5(3.7-5.3)$ & \\
\hline Values are present & $396(234-559)$ & \\
\hline
\end{tabular}

Values are presented as percentages, mean \pm standard deviations (SD) or median (interquartile range).

$* p<0.05$.

Table 2. Correlation coefficients for the association of zonulin in controls and OSA patients.

\begin{tabular}{|c|c|c|c|c|}
\hline & \multicolumn{2}{|c|}{ Controls } & \multicolumn{2}{|c|}{ OSA } \\
\hline & $\mathbf{R}$ & $p$ & $\mathbf{R}$ & $p$ \\
\hline Waist circumference $(\mathrm{cm})$ & 0.382 & 0.04 & 0.442 & 0.004 \\
\hline Glucose (mg/dL) & 0.343 & 0.035 & 0.321 & 0.04 \\
\hline Insulin $(\mu \mathrm{UI} / \mathrm{mL})$ & 0.328 & 0.041 & 0.351 & 0.03 \\
\hline Cholesterol (mg/dL) & 0.135 & 0.419 & 0.397 & 0.011 \\
\hline Mean $\mathrm{SaO}_{2}(\%)$ & -0.315 & 0.061 & -0.378 & 0.019 \\
\hline $\operatorname{ALT}(\mathrm{U} / \mathrm{L})$ & 0.001 & 0.996 & 0.484 & 0.002 \\
\hline $\mathrm{AST}(\mathrm{U} / \mathrm{L})$ & -0.006 & 0.972 & 0.426 & 0.006 \\
\hline GGT (U/L) & 0.158 & 0.343 & 0.444 & 0.004 \\
\hline hs-CRP (mg/L) & 0.320 & 0.054 & 0.372 & 0.02 \\
\hline
\end{tabular}

Comment [JM18]: As before
Comment [JM19]: As before
Comment [JM20]: As before
Comment [JM21]: As before

Table 3. Multivariate analysis of factors associated with zonulin in OSA patients 


\begin{tabular}{|lccc|}
\hline & $\boldsymbol{\beta}$ & $\mathbf{9 5 \%} \mathbf{C I}(\boldsymbol{\beta})$ & $\boldsymbol{p}$ \\
$\mathrm{Mean} \mathrm{SaO}_{2}(\%)$ & 0.008 & $-0.049-0.034$ & 0.711 \\
Cholesterol (mg/dL) & 0.001 & $-0.001-0.003$ & 0.267 \\
$\mathrm{ALT}(\mathrm{U} / \mathrm{L})$ & 0.014 & $0.001-0.028$ & 0.04 \\
$\mathrm{AST}(\mathrm{U} / \mathrm{L})$ & 0.02 & $0.002-0.037$ & 0.04 \\
$\mathrm{GGT}(\mathrm{U} / \mathrm{L})$ & 0.01 & $-0.005-0.008$ & 0.681 \\
hs-CRP (mg/L) & 0.075 & $0.008-0.158$ & 0.046 \\
\hline
\end{tabular}

Comment [JM22]: As other tables 